\title{
Recherche comparative internationale à la base de trois méthodes de LSP
}

\section{Martine Verjans}

\section{OpenEdition}

\section{Journals}

Édition électronique

URL : http://journals.openedition.org/asp/2956

DOI : 10.4000/asp.2956

ISSN : 2108-6354

\section{Éditeur}

Groupe d'étude et de recherche en anglais de spécialité

\section{Édition imprimée}

Date de publication : 1 décembre 1997

Pagination : 27-39

ISSN : 1246-8185

\section{Référence électronique}

Martine Verjans, "Recherche comparative internationale à la base de trois méthodes de LSP », ASp

[En ligne], 15-18 | 1997, mis en ligne le 27 avril 2012, consulté le 19 avril 2019. URL : http:// journals.openedition.org/asp/2956 ; DOI : 10.4000/asp.2956

Ce document a été généré automatiquement le 19 avril 2019

Tous droits réservés 


\title{
Recherche comparative internationale à la base de trois méthodes de LSP
}

\author{
Martine Verjans
}

\section{Justification de la recherche}

1 La recherche comparative internationale Linguaplan Limburg 1993-1997¹ ayant mené au développement de trois méthodes de langues de spécialité (LSP), trouve ses origines dans deux constatations : le caractère essentiellement exportateur de l'activité économique flamande d'une part et la pénurie de méthodes d'apprentissage ciblées de langue étrangère $(\mathrm{LE})^{2}$ pour des publics professionnels d'autre part.

2 L'économie flamande ${ }^{3}$ repose essentiellement sur un très dense tissu de PME-PMI ${ }^{4}$ à effectif réduit ${ }^{5}$ réalisant un pourcentage important ${ }^{6}$ de leur chiffre d'affaires grâce à l'exportation. Franchir des frontières nationales n'implique pas forcément la confrontation à une autre langue, mais la situation géographique spécifique ${ }^{7}$ des entreprises flamandes inclut tout naturellement un contact allophone, constatation d'autant plus valable pour le Limbourg. ${ }^{8}$ Obstacle ou défi ?

3 Malgré le nombre toujours croissant des centres de formation en communication allophone et en dépit des multiples initiatives d'organismes en tout genre (patronat flamand, gouvernement, $\mathrm{VDAB}^{9}$ ), entrepreneurs et formateurs ne se disent que rarement satisfaits des résultats des formations suivies. Comment expliquer que, des deux côtés, de sérieux efforts soutenus n'aboutissent pas au rendement tellement urgent?

Il s'avère que trop souvent, les méthodes d'apprentissage de LE destinées tant aux publics professionnels qu'aux élèves de la formation initiale (surtout professionnel et technique) sont doublement inadaptées : leur contenu ne correspond pas aux besoins professionnels (futurs) des apprenants, et leur démarche didactique ne prend pas suffisamment en compte les spécificités du groupe cible. Les centres de ressources ne fournissent pas non 
plus les solutions requises: ordinairement ils ne présentent que des documents authentiques bruts et ils sont le plus souvent liés à l'apprentissage en autonomie complète ou en semi-autonomie - dernière parade de nombre d'organismes. Plusieurs facteurs (psycholinguistiques et autres) sont probablement la cause de la rentabilité médiocre de ce genre de formations. Une dialectique rigoureuse à ce sujet nous mènerait trop loin puisqu'elle n'entre pas dans les objectifs du présent article, mais elle se justifierait certainement. Les "autoroutes de l'information", dernière nouveauté technologique et si alléchantes qu'elles soient du point de vue de la motivation (elles pourront bien servir de carotte...), ne permettent malheureusement (toujours) pas l'entrainement à la production orale ni l'évaluation de cette compétence combien importante dans la plupart des milieux professionnels.

Pour aider à faire face à ces multiples défaillances, l'unité de recherche en linguistique appliquée du Centre universitaire du Limbourg (LUC-CTTL) a monté le projet mentionné. Cette recherche comparative se voulait à échelle internationale afin de pouvoir découvrir les différences éventuelles entre les pays concernés quant aux besoins de communication professionnelle en LE, en vue de l'objectif final, à savoir la réalisation de modules répondant aux besoins identifiés, utilisables dans l'enseignement présentiel ou en semiautonomie (avec tutorat). Ils devraient conduire au plus vite à la fixation d'automatismes de communication professionnelle en évitant les explications grammaticales et toute forme de métalangue.

\section{Des contenus adaptés aux besoins professionnels (futurs) en matière de communication en LE}

$6 \quad$ Le principe de base de Linguaplan Limburg 1993 - 1997 était de puiser toute information dans les différents secteurs économiques auprès des personnes mêmes en poste. Cette approche nous paraît la seule fournissant des garanties d'authenticité et de fonctionnalité aux contenus d'une méthode conçue pour des publics à besoins précis et délimités. D'ailleurs, le projet Linguaplan Limburg s'était fixé comme objectif final de développer des modules de communication en LE afin de satisfaire les réels besoins des utilisateurs. À cette fin, un consortium a été composé regroupant des centres de recherche en linguistique appliquée ${ }^{10}$ ainsi que des organisations professionnelles et patronales ${ }^{11}$ de cinq pays de l'UE.

Cette recherche comparative internationale s'est déroulée en trois phases.

8 La première phase, plutôt générale et essentiellement quantitative, a fourni l'inventaire des besoins en matière de communication en LE, toutes fonctions et toutes activités économiques confondues, et ce au niveau transnational, à savoir des pays partenaires du projet. Une réponse à plusieurs questions s'imposait :

- dans quels secteurs et pour quelles fonctions les besoins en LE s'avèrent-ils les plus urgents?

- pour quelles langues ou, en d'autres termes, quelle est la hiérarchie des LE?

- quel est le mode de communication privilégié (téléphone, télécopie...) ?

- quelle est la proportion de langue générale et de langue de spécialité (LSP) ?

- existe-t-il une corrélation entre la maîtrise d'une LE et le chiffre d'affaires réalisé dans cette langue?

- quelle est l'importance de la connaissance des LE pour le salarié (recrutement, carrière) ? 
- le cas échéant, faute de compétence langagière, comment l'entreprise essaie-t-elle de «se débrouiller » (recours à la sous-traitance et coûts afférents) ?

9 Une enquête écrite fut diffusée auprès de 848 PME-PMI, échantillon représentatif correspondant aux codes $\mathrm{NACE}^{12}$ ou $\mathrm{APE}^{13}$ et à la taille des entreprises établies dans les régions examinées des pays concernés. L'interprétation des résultats des tris à plat et des croisements significatifs a été soumise à l'avis de "témoins privilégiés " des secteurs enquêtés et ce d'après les principes de l'audit d'entreprise. L'analyse des données s'est limitée pourtant aux seuls secteurs secondaire et tertiaire, le secteur primaire (code NACE 0 , comprenant le domaine des activités productrices de matières non transformées) n'ayant fourni que quelques observations.

10 À titre illustratif ${ }^{14}$, voici quelques résultats (en valeurs absolues ou en fréquences relatives) comparant la province du Limbourg (B) avec la région d'Anjou (F $)^{15}$. Quant à l'interprétation de ces constatations ou à l'ensemble des résultats, nous renvoyons au rapport Linguaplan Limburg - Phase 1 (voir références bibliographiques).

\section{Hiérarchie des LE et leur relation réciproque}

- B : le français s'avère le plus important (coefficient 6), suivi par l'allemand (3) et l'anglais (2), l'italien et l'espagnol ;

- F : l'anglais domine nettement (coefficient 6), suivi par l'allemand (3), puis par le castillan et le néerlandais (2), l'italien, le portugais, l'arabe et le catalan.

\section{Fréquence des contacts allophones}

- B : plus de trois PME-PMI sur quatre prétendent avoir au moins un contact allophone par jour ; plus l'entreprise est grande (plus de dix salariés), plus elle a de tels contacts ;

- F : la moitié des entreprises n'utilise que le français ; seulement neuf des 265 entreprises ont (en moyenne) un contact allophone par jour.

\section{Chiffre d'affaires (CA)}

- B : plus d'un quart réalise plus de $20 \%$ de son CA en français et plus de $10 \%$ en allemand (ce qui confirme la hiérarchie);

- F : si l'entreprise réalise une partie de son C.A. dans une langue étrangère, celui-ci ne dépasse que rarement les $5 \%{ }^{16}$.

\section{Influence sur la carrière}

- B : pour 86,2 \% des PME-PMI sondées, la maîtrise d'une (et de préférence deux, voire trois) LE représente un atout majeur ;

- F : seulement $30,1 \%$ en font un critère de sélection.

\section{Initiatives afin d'améliorer la connaissance des LE du personnel ${ }^{17}$}

- B : seulement 56,5 \% des PME-PMI où l'on estime que les LE influent sur la carrière, prennent des initiatives ;

- F : seuls 5,6 \% des entreprises interrogées font des propositions (cours à l'extérieur).

\section{LSP versus langue générale ; oral versus écrit}

- B : les besoins se situent aussi bien au niveau de la langue de spécialité qu'au niveau de la langue générale, quoique cette dernière soit considérée comme plus importante, sauf pour les fonctions techniques ${ }^{18}$;

- F : pour les entreprises exprimant des besoins en LE, une légère prédominance de la langue générale se manifeste $(35,33 \%)$ par rapport à la langue de spécialité $(21 \%)$, et ce plutôt au niveau des directions.

Surtout en Belgique, où elles constituent plus de $25 \%$ des tâches, les activités en LE se font à l'oral (direction, fonctions techniques supérieures, fonctions orientées vers 
l'extérieur), voire seulement à l'oral pour les fonctions techniques inférieures, tandis qu'au niveau de l'administration inférieure, oral et écrit se tiennent en équilibre.

\section{Appel à un service de traduction}

- B : 190 des 205 entreprises enquêtées s'adressent à des traducteurs extérieurs ${ }^{19}$. Il faut néanmoins relativiser ces résultats puisqu'il s'agit essentiellement d'aide au niveau de l'écrit, celui-ci ne représentant pourtant que $13 \%$ de la communication d'affaires ;

- F : 9,7\% des entreprises de l'échantillon font appel à un service extérieur (représentant seulement $7000 \mathrm{FRF}$ par an en moyenne), tandis que $15 \%$ disposent d'un service intégré (anglais 7,8 \%, allemand 4,1\%, espagnol/castillan 1,5\%).

\section{Échecs à la suite d'un manque de connaissances en LE}

- B : $25 \%$ des interrogés avouent avoir dû refuser des ordres ou l'échec de contacts (avec des répercussions directes sur le chiffre d'affaires !) ;

- F : 93,7\% prétendent n'avoir jamais (!) eu de difficultés.

12 Les principes méthodologiques de cette première partie de la recherche forment aussi la base de la deuxième phase à caractère plutôt qualitatif. L'objectif a été double: la constitution et l'analyse de corpus oraux (entretiens téléphoniques, réunions, face à face...) et écrits (lettres, télécopies, autres documents...) liés à plusieurs fonctions, et, ensuite, la composition des profils langagiers correspondants. Le dépouillement des corpus a été effectué à plusieurs niveaux: lexique, micro- et macrocombinaison (collocations et discours), nuance de sens et registre.

13 Le choix des six profils retenus ${ }^{20}$ résulte directement de la première phase de la recherche, à savoir les secteurs et fonctions éprouvant les plus grands problèmes de communication allophone.

14 La composition des profils a requis une préparation minutieuse, à savoir la rédaction d'un référentiel général à partir de la littérature disponible (parfois sommaire). Ces grilles d'entretien, d'abord prévalidées par un spécialiste du secteur concerné, ont servi de base à tout entretien, quelle que soit sa nature, avec des "témoins privilégiés »: remueméninges avec des personnes en poste, tant horizontal (parcourir toutes les activités communicatives d'une année avec mention de leur fréquence et de leur importance) que vertical (une journée type du matin au soir), entretiens en face à face avec le supérieur hiérarchique et ensuite avec une ou plusieurs personnes en poste. La comparaison des informations obtenues par ces différentes voies a conduit aux profils authentiques de fonction.

Chacun de ces profils linguistiques comprend l'inventaire détaillé des situations de communication et des actes de parole propres à la fonction concernée. À ces données ont été ajoutées des informations concernant le média utilisé, la fréquence ( $\mathrm{I}=$ au moins une fois par jour... III = de temps en temps seulement) et l'importance de l'acte $(1=$ très important, 2 = important, 3 = moins important) ainsi que son caractère réceptif $(\mathrm{R})$ ou productif (P), écrit (é) ou oral (o). De plus, l'analyse fréquentielle de corpus a permis d'enrichir chaque profil des composantes langagières les plus représentatives de la fonction examinée.

Vu le caractère européen de Linguaplan Limburg 1993-1997, tous les profils étant établis par une seule équipe du projet ont dû être validés à échelle transnationale. Ainsi, ces inventaires initiaux ont été soumis pour validation à un partenaire étranger. Il a surtout fallu déterminer si les différences observées révélaient un oubli ou plutôt une différence nationale. Les profils définitifs résultent donc du travail de binômes de recherche, dont 
chaque pôle se situe dans un autre pays partenaire du projet. Le cas échéant, la distinction entre le profil commun, donc international et les spécificités nationales a été accentuée par la mise en page.

À titre d'exemple, un extrait du profil «conducteur de poids lourd $»^{21}$ a été ajouté en annexe ${ }^{22}$.

À nos yeux, ces profils linguistiques détaillés, enrichis des composantes langagières issues de l'analyse de corpus, sont indispensables à la réalisation des modules ciblés de LE, objectif de la troisième phase de Linguaplan Limburg 1993 - 1997. Les ensembles d'apprentissage de communication en LE conçus dans le cadre de cette recherche sont tous multimédias (manuel de l'apprenant, guide, cassette audio et/ou vidéo, logiciel) ${ }^{23}$. Ils constituent la série intitulée Praktische Communicatie Vreemde Talen ${ }^{24}$ qui, en raison de ses contenus spécifiques ainsi que de son approche méthodologique, peut se vanter de fournir un apport original à la formation en LE de spécialité sans négliger pourtant la langue générale.

Les inventaires de communication professionnelle se sont avérés d'autant plus opérationnels qu'ils ont révélé un tronc commun d'actes de parole et de situations de communication, pour tous les secteurs examinés. Cette constatation a conduit aux modules des Actes de Base des Professions développés en quatre langues sources (néerlandais, français, espagnol, anglais) avec quatre langues cibles (français, espagnol, anglais, allemand).

\section{Principes méthodologiques des modules d'apprentissage}

Toute méthode efficace, qui prétend apporter une réelle solution à des besoins précis en communication liés à une fonction, doit garantir l'authenticité langagière. Grâce aux profils élaborés et à l'analyse (fréquentielle) de corpus oraux et écrits, les éléments de langue utilisés effectivement (et fréquemment) par des locuteurs natifs « en poste » conduisent à un ou plusieurs dialogues qui forment le point de départ de chaque dossier des modules développés. Ces dialogues réunissent des extraits de plusieurs enregistrements authentiques.

21 En plus, les exercices sont basés sur les contenus et les scénarios de ces dialogues enregistrés et transcrits. Ils sont donc tout à fait authentiques, quoiqu'une différence de degré de difficulté ait été poursuivie au fil des exercices. Les répliques du natif allophone sont toujours restées inchangées et elles semblent par conséquent plus « difficiles » que celles de l'apprenant, se limitant à l'essentiel du message à communiquer dans un langage simplifié, mais pourtant authentique. En effet, à quoi bon simplifier artificiellement l'entrainement de la compréhension auditive ? Rien de plus navrant, voire frustrant que de constater qu'on ne comprend toujours pas son interlocuteur allophone et ce après avoir parcouru toute une méthode de communication en LE! Pour les apprenants plus avancés, des variantes couramment utilisées par les natifs sont proposées sans être systématiquement insérées dans les multiples exercices présentés.

22 Un deuxième principe de base de l'approche méthodologique est celui de la progression ou de la progressivité. En passant par plusieurs phases se chevauchant partiellement et cela conformément au processus d'apprentissage naturel, un riche éventail d'exercices 
caractérisant chacun des volumes de la série Praktische Communicatie Vreemde Talen mènera l'utilisateur à des automatismes de communication en LE.

Réception, reproduction, reformulation, production guidée..., des étapes obligées pour aboutir à ces automatismes et, par là, à la production libre, aboutissement du transfert réussi.

Grâce à ces principes testés auprès de plusieurs groupes cibles, tout apprenant s'appropriera une communication naturelle, essentiellement axée sur des compétences orales ${ }^{25}$. Sans recours au métalangage et avec un minimum d'explications grammaticales, les modules développés se font forts d'arriver à des résultats certains. Par leur utilité immédiate, ils motiveront même les apprenants (en formation initiale ou continue) moins doués et ne décevront pas les professionnels aux besoins précis et urgents (le temps c'est de l'argent) en matière de communication en LE.

Ainsi, le projet Linguaplan Limburg 1993-1997, par ses bases rigoureusement scientifiques et par son approche méthodologique originale, espère contribuer à résoudre les problèmes de communication en LE qui handicapent si souvent les milieux professionnels.

\section{BIBLIOGRAPHIE}

Gewestelijke Ontwikkelings Maatschappij - Limburgse Economische Raad (GOM-LER). 1996. Jaarverslag. Peeters, L. et al. Recherche en cours, LUC-TEW en coopération avec GOM-Limburg. Diepenbeek, Belgique.

Statistiek Buitenlandse Handel. 1996. Bruxelles.

Verjans, M. \& W. Clijsters et al. 1994. Linguaplan Limburg - Phase 1: Rapport des audits régionaux. Diepenbeek, Belgique : LUC-CTTL.

Verjans, M. \& W. Clijsters, et al. 1995. Linguaplan Limburg - Phase 2: Rapport 1994 - Profils linguistiques. Diepenbeek, Belgique : LUC-CTTL.

\section{Bibliographie complémentaire}

Bertolacci, M. Vreemde-talenbehoeften van KMO's in Nederlands Limburg. 1995. Diepenbeek, Belgique : LUC-TEW.

Clijsters, W. 1990. Mille lettres d'affaires en chiffres. Paris et Genève : Champion-Slatkine.

Clijsters, W. et al.1993. Les médias dans les affaires - Intercom S.A.. Anvers, Belgique : Plantyn.

Royall, F. et al. 1992. « The Rip Van Winkle syndrome: When will Irish wake up to the importance of foreign languages skills ». Technology Ireland 24/4, 42-44.

Royall, F. et al. 1993a. « Language needs analysis ». Industry and Higher Education 7/1, 151-52.

Royall, F. et al. 1993b. « The status of languages in export-oriented companies: A survey of the Irish scene ». ABLA-Papers, Association belge de linguistique appliquée 15, Taal en Bedrijf, 97-106.

Royall, F. 1994a. « Languages and specialisms: Bridging the gap ». Languages for the International Scientist. Londres, 131-142. 
Royall, F. 1994b. « Le développement d'un logiciel pour standardistes de PME/PMI ». CEL-INFO 18, 2.

Van Baelen, E. 1991. Vreemde-talenkennis in KMO's: een onderzoek naar behoeften en gebruik in het arrondissement Turnhout. Diepenbeek, Belgique : LUC-TEW.

Van der Velden, H. 1988. Vreemde-talenbehoeften in Limburgse KMO's. Diepenbeek, Belgique : EHLLUC.

Verjans, M. \& W. Clijsters. 1992. Tous Azimuts. Lier, Belgique : Van In.

Verjans, M. \& W. Clijsters. 1993. « Les PME devant le défi des langues étrangères ». ABLA-Papers, Association belge de linguistique appliquée 15, Taal en Bedrijf, 37-44.

Verjans, M. \& P. D’hondt. 1993. «Le ‘savoir-faire' de la production orale libre ». Romaniac 52-3, 1993, p. 17-35 et ERIC, Clearinghouse on Languages and Linguistics, Washington.

Verjans, M. \& W. Clijsters. « La quadrature du cercle, comment combler les lacunes dans la compétence en langue(s) étrangère(s) des publics professionnels? » Les cahiers de l'APLIUT, Paris, 1994 et ERIC, Clearinghouse on Languages and Linguistics, Washington.

Verjans, M. \& W. Clijsters. « Les langues et les entreprises » Actes du congrès de la section de français de 'Vereniging voor Levende Talen', Noordwijkerhout (NL) : 1995, pp. 73-76

Verjans, M. \& W. Clijsters. «La recherche au service du concepteur et rédacteur d'une méthode de langue de spécialité avec des exemples du français des affaires », Issues in Languages for Specific Purposes, Theoretical Approaches and Practical Applications, Limerick (IRL) : University of Limerick Press, 1996, pp. 105-112.

Verjans, M. \& W. Clijsters et al. Linguaplan Limburg - Fase 1: Rapport van de regionale audit, Limburg, Diepenbeek (B) : LUC-CTTL, 1994.

Verjans, M. \& W. Clijsters et al. Communication-Services-Recrutement, Antwerpen (B) : Plantyn, 1995.

Verjans, M. \& W. Clijsters et al. Mots-clefs des affaires, Paris : Foucher, 1996.

\section{ANNEXES}

Annexe 1 (GOM-LER, Statistiek Buitenlandse Handel, 1996) Taille des entreprises flamandes $(\mathrm{N}=143421)$ par nombre de salariés

\begin{tabular}{|l|l|}
\hline Effectif & Nombre d'entreprises (tous secteurs confondus) \\
\hline $1-9$ & 116221 \\
\hline $10-49$ & 21541 \\
\hline $50-199$ & 4477 \\
\hline $200-499$ & 860 \\
\hline $500-999$ & 211 \\
\hline$>1000$ & 111 \\
\hline
\end{tabular}


Annexe 2 (Verjans et al. 1995 : 25-26) Extrait du profil « conducteur de poids lourd »

3. Contacts avec le client

3.1 Charger I

3.1.1 se manifester I Po

3.1.1.1 se manifester au nom de sa propre firme ou au nom du client

3.1.1.2 à quel quai / sabord on doit se présenter Po Ro

3.1.1.3 destination de la charge Po Ro

3.1.2 pesage III

3.1.2.1 comprendre que le camion doit être pesé Ro

3.1.2.2 demander où se trouve le pont bascule Po

3.1.3 charger I

3.1.3.1 désigner les outils (transpalette/chariot élévateur/plaque de roulage...)

1 Po

3.1.3.2 comprendre les manipulations/ en charger $\mathrm{qqn}^{26}$ (couvrir/pousser...)

2 Po Ro

3.1.3.3 faire respecter des directives ${ }^{27} 1 \mathrm{Po}$

3.1.3.4 avertir d'un danger (demander de s'éloigner/invoquer la défense de fumer...) 1 Po

3.1.4 contrôler

3.1.4.1 comprendre 1 Ré

3.1.4.1.1 étiquettes

3.1.4.2 CMR / « packing list » - liste de colisage ${ }^{28}$

3.1.5 refuser de partir ${ }^{29} 1$ Po

3.1.5.1 $\ldots$

3.1.6 partir I

3.1.6.1 CMR 1

3.1.6.1.1 demander de remplir la CMR 1 Po

3.1.6.1.2 faire des réserves, commenter et comprendre 1 Poé Roé

3.1.6.1.3

3.1.6.2 véhicule (véhicule ouvert/non ouvert/une bâche/convenu avec l'expéditeur...)

3.1.6.3 emballage (sans emballage/emballage défectueux/insuffisant/ non conforme...)

3.1.6.4 colis

3.1.6.4.1 $\ldots$

3.1.6.5 état des marchandises offertes/reçues/réceptionnées

3.1.6.5.1 endommagées/avariées... 
3.1.6.5.2 $\ldots$

3.1.6.6 manutention, chargement, arrimage, déchargement

3.1.6.6.1 ...

3.1.6.6.2 palette jetable, tandis que CMR mentionne europalette

3.1.6.6.3 demander le cachet de la firme 3 Po

Annexe 3 (Verjans et al. 1994 : 114) PME-PMI angevines

Pourcentage du chiffre d'affaires (CA) réalisé dans une autre langue que la langue véhiculaire de l'entreprise

\begin{tabular}{|l|l|l|l|l|l|}
\hline CA & $0-5 \%$ & $6-10 \%$ & $11-20 \%$ & $21-50 \%$ & aucune idée \\
\hline
\end{tabular}

valeurs absolues (nombre d'entreprises)+fréquences relatives LANGUE

\begin{tabular}{|c|c|c|c|c|c|}
\hline anglais & 111 & 10 & 3 & - & 142 \\
\hline & $41,7 \%$ & $3,8 \%$ & $1,1 \%$ & - & $53,4 \%$ \\
\hline \multirow[t]{2}{*}{ allemand } & 40 & - & 1 & 2 & 223 \\
\hline & $15 \%$ & - & $0,4 \%$ & $0,8 \%$ & $83,8 \%$ \\
\hline \multirow[t]{2}{*}{ castillan } & 15 & 1 & - & - & 250 \\
\hline & $5,6 \%$ & $0,4 \%$ & - & - & $94 \%$ \\
\hline \multirow[t]{2}{*}{ catalan } & 2 & - & - & - & 264 \\
\hline & $0,8 \%$ & - & - & - & $99,2 \%$ \\
\hline \multirow[t]{2}{*}{ italien } & 10 & 4 & - & - & 252 \\
\hline & $3,8 \%$ & $1,5 \%$ & - & - & $94,7 \%$ \\
\hline \multirow[t]{2}{*}{ portugais } & 6 & - & - & - & 260 \\
\hline & $2,3 \%$ & - & - & - & $97,7 \%$ \\
\hline \multirow[t]{2}{*}{ néerlandais } & 14 & 2 & - & 1 & 249 \\
\hline & $5,3 \%$ & $0,8 \%$ & - & $0,4 \%$ & $93,5 \%$ \\
\hline \multirow[t]{2}{*}{ arabe } & 3 & 1 & - & - & 262 \\
\hline & $1,1 \%$ & $0,4 \%$ & - & - & $98,5 \%$ \\
\hline autre & 7 & - & - & 1 & 258 \\
\hline
\end{tabular}




\begin{tabular}{|l|l|l|l|l|l|}
\hline & $2,6 \%$ & - & - & $0,4 \%$ & $97 \%$ \\
\hline
\end{tabular}

\section{Annexe 4 (Verjans et al. 1994, 72) Nature des langues étrangères utilisées}

$L G=$ pourcentage des entreprises de l'échantillon qui prétendent que dans la fonction en question il faut une bonne maîtrise de la langue générale

LSP = pourcentage des entreprises de l'échantillon qui prétendent que dans la fonction en question il faut une bonne maîtrise de la langue de spécialité

\begin{tabular}{|c|c|c|c|c|}
\hline & \multicolumn{2}{|c|}{ Direction } & \multicolumn{2}{|c|}{$\begin{array}{c}\text { Fonctions techniques } \\
\text { supérieures }\end{array}$} \\
\hline & LG & LSP & LG & LSP \\
\hline français & 81,3 & 72,5 & 42,4 & 58,5 \\
\hline allemand & 61,1 & 54,9 & 32,1 & 41,9 \\
\hline anglais & 64,2 & 53,8 & 36,7 & 50,7 \\
\hline espagnol & 4,1 & 1,5 & 0 & 0,5 \\
\hline italien & 2,5 & 3,1 & 1 & 1 \\
\hline portugais & 0 & 0,5 & 0 & 0 \\
\hline \multirow[t]{3}{*}{ autre } & 0,5 & 0 & 0 & 0,5 \\
\hline & \multicolumn{2}{|c|}{$\begin{array}{c}\text { Fonctions techniques } \\
\text { inférieures }\end{array}$} & \multicolumn{2}{|c|}{$\begin{array}{l}\text { Fonctions administratives } \\
\text { inférieures }\end{array}$} \\
\hline & LG & LSP & LG & LSP \\
\hline français & 24,3 & 35,2 & 51,8 & 38,3 \\
\hline allemand & 14,5 & 20,2 & 30 & 21,7 \\
\hline anglais & 18,1 & 25,9 & 33,6 & 23,8 \\
\hline espagnol & 0 & 0 & 0,5 & 0 \\
\hline italien & 0,5 & 0,5 & 0,5 & 0 \\
\hline \multirow[t]{3}{*}{ autre } & 0,5 & 0 & 0 & 0 \\
\hline & \multicolumn{2}{|c|}{ Fonctions extérieures } & & \\
\hline & LG & LSP & & \\
\hline français & 71,5 & 52,3 & & \\
\hline allemand & 55,4 & 37,8 & & \\
\hline anglais & 54,9 & 35,7 & & \\
\hline espagnol & 2 & 1 & & \\
\hline italien & 1,5 & 1,5 & & \\
\hline portugais & 0,5 & 0 & & \\
\hline autre & 0,5 & 0 & & \\
\hline
\end{tabular}

Annexe 5 (Verjans et al. 1994 : 83) Appel à un service de traduction

\begin{tabular}{|l|l|l|}
\hline Type d'aide & $\begin{array}{l}\text { Nombre de PME-PMI ayant répondu à la } \\
\text { question }\end{array}$ & Pourcentage \\
\hline 1 traduction contrats & 55 & $35,4 \%$ \\
\hline 2 traduction technique & 51 & $32,9 \%$ \\
\hline 3 traduction publicitaire & 45 & $29 \%$ \\
\hline $\begin{array}{l}4 \quad \text { traduction de lettres, } \\
\text { formules... }\end{array}$ & 29 & $18,7 \%$ \\
\hline $\begin{array}{l}5 \quad \text { interprétariat lors de } \\
\text { négociations }\end{array}$ & 11 & $7 \%$ \\
\hline $6 .$. & & \\
\hline
\end{tabular}




\section{NOTES}

1. Voir références bibliographiques.

2. Langues étrangères

3. La Flandre = la partie néerlandophone de la Belgique = Nord de la Belgique $\left(13152 \mathrm{~km}^{2}\right)$

4. Petites et Moyennes Entreprises - Petites et Moyennes Industries

5. Voir annexe 1

6. À titre d'exemple quelques données (1993) : volume de production (en BEF) de la Flandre : 7 729,8 milliards (Peters, recherche en cours) dont 2881,3 exportés vers l'étranger (GOM-LER 1996). Remarquons que l'exportation ne constitue qu'une partie des flux économiques d'un pays.

7. En Belgique, il y a trois communautés (recensement de 1994: néerlandophone : 5847022 personnes, francophone : 3304539 personnes, et germanophone : 68741 personnes, à l'exclusion de Bruxelles [949 070 personnes] dont les données ne sont pas disponibles), d'où trois langues officielles, à savoir le néerlandais, le français et l'allemand. La France, les Pays-Bas (la langue de négociation y est souvent l'anglais) et l'Allemagne, pays voisins, représentent en plus les premiers partenaires commerciaux de la Flandre (exportation en 1993: vers la France : 426,8 milliards de BEF, vers les Pays-Bas : 385,5 milliards de BEF et vers l'Allemagne : 554,3 milliards de $\mathrm{BEF}$ ). S'ajoute le flux commercial vers la Wallonie et vers la partie germanophone de la Belgique (données indisponibles).

8. Le Limbourg $\left(2425 \mathrm{~km}^{2}\right)$, situé dans le nord-est de la Flandre, est entouré par les principales régions industrielles de l'Europe occidentale : la Randstad Holland avec Amsterdam, Rotterdam et La Haye, la région allemande de la Ruhr, la zone industrielle du Nord de la France et la région du sud-est de l'Angleterre. Elle se trouve à proximité de la capitale économique flamande (Anvers, $80 \mathrm{~km}$ ), belgo-européenne (Bruxelles, $77 \mathrm{~km}$ ) et des bassins industriels wallons. Son centre universitaire est situé à $37 \mathrm{~km}$ de Liège (Wallonie) et à $53 \mathrm{~km}$ d'Aix-la-Chapelle (Allemagne).

9. Comparable avec l'ANPE, bien que le VDAB (Vlaamse Dienst voor Arbeids Bemiddeling) organise aussi des formations

10. Centrum voor Toegepaste Taalkunde en Literatuurstudie - Limburgs Universitair Centrum (LUC-CTTL Diepenbeek), les unités respectives de recherche en linguistique appliquée des universités de Dunkerque (F), de Limerick (IRL) et de Valladolid (E), de l'ESSCA (Ecole Supérieure des Sciences Commerciales d'Angers, F) et LRSU (Language Research and Services Unit, Limerick, IRL).

11. Essentiellement les Chambres de Commerce et d'Industrie locales et régionales des partenaires du projet de recherche.

12. Classification européenne des activités économiques

13. Activité Principale Exercée (souvent utilisée en France au lieu du code NACE)

14. Pour l'étude exhaustive : voir le rapport intégral 1994 (voir références bibliographiques)

15. Nous sommes partis de l'hypothèse que la situation géographique aurait une influence sur les résultats rassemblés dans la première phase de la recherche. Ainsi nous avons voulu montrer la corrélation entre maîtrise de LE et croissance de l'entreprise (donc augmentation de ses effectifs), dont certains patrons ne sont nullement conscients.

16. Voir annexe 3.

17. Une explication s'impose : d'après les « témoins privilégiés » (B et F), les cours sur le marché sont trop généraux, trop chronophages et trop coûteux.

18. Voir annexe 4.

19. Voir annexe 5 - La sous-traitance s'avère nettement moins coûteuse qu'un service propre. 
20. Transitaire, dispatcher et conducteur de poids lourd (transport international); accueil dans les bureaux d'information, réception des hôtels et restaurants (tourisme); responsable d'agence (immobilier)

21. Pour cette fonction, il n'y avait pas de différences nationales.

22. Voir annexe 2 .

23. La composition du consortium Linguaplan Limburg 1993 - 1997 a permis de réaliser ces modules en binôme : langue source et langue cible. De ces ensembles destinés à des fonctions particulières sont déjà achevés : transport international: transitaire (néerlandais - français et français néerlandais), tourisme : fonction d'accueil (néerlandais - espagnol).

24. Éditions Wolters - Plantyn, Deurne-Anvers, Belgique

25. Voir rapport Linguaplan Limburg - phase 1 : l'oral constitue plus de $80 \%$ de la communication d'affaires.

26. Selon les dispositions de l'accord CMR, l'expéditeur doit s'occuper du chargement et le destinataire du déchargement. Le chauffeur est responsable de l'arrimage des marchandises. Le chauffeur ne peut pas participer aux opérations de chargement et de déchargement. Pourtant, dans la pratique il donnera toujours un coup de main.

27. Très important. Par exemple, demander un câble supplémentaire quand les marchandises doivent être déchargées en utilisant plus de deux câbles (les palettes fléchissent). Refuser de laisser un chariot élévateur trop lourd dans le camion.

28. Important pour la répartition du poids et pour l'arrimage.

29. Faire des réserves, commenter et comprendre les réactions. Certaines entreprises de transport refusent de partir si les réserves n'ont pas été mentionnées.

\section{RÉSUMÉS}

Linguaplan Limburg, recherche comparative internationale, a d'abord dressé l'inventaire des problèmes d'ordre linguistique de 1000 PME-PMI. Cette première partie quantitative fut suivie par une phase axée sur une analyse qualitative à double objectif: l'établissement de profils langagiers détaillés de certains métiers-professions résultant du susdit inventaire d'une part, la constitution et l'analyse de corpus écrits et oraux d'autre part. Cette exploration qualitative a constitué la base de la troisième phase, celle de la conception et de l'élaboration de modules langagiers d'apprentissage ciblés et multimédias, de la taille la plus opérationnelle possible, et se prêtant à l'auto-apprentissage.

The principal aim of Linguaplan Limburg was a large comparative and international research. It has, in a first stage, summed up a great number of linguistic problems (in about 1000 SMEs) only to focus in a second stage on an exclusively qualitative study. This qualitative study deals on the one hand with defining the language profiles specific to a number of professions and gives on the other hand a written and oral corpus including its analysis. This study was the basis for the creation of language learning methods concentrating both on professional needs and multimedia. Being as user-friendly as possible, the methods also aim home study. 
INDEX

Mots-clés : analyse de corpus, besoins, communication professionnelle, didactique des langues, langue de spécialité, profil langagier de fonctions

Keywords : analysis of corpus, foreign language didactics, language for specific purposes, need, professional communication, business language profile

\section{AUTEUR}

\section{MARTINE VERJANS}

